This is an Open Access article, distributed under the terms of the Creative Commons Attribution licence (http://creativecommons.org/licenses/by/4.0/), which permits unrestricted re-use, distribution, and reproduction in any medium, provided the original work is properly cited.

\title{
Optical shaping of gas targets for laser-plasma ion sources
}

\author{
N. P. Dover ${ }^{1} \dagger$, N. Cook $^{2} \ddagger$, O. Tresca ${ }^{3}$, O. Ettlinger ${ }^{1}$, C. Maharjan ${ }^{2}$, \\ M. N. Polyanskiy ${ }^{3}$, P. Shkolnikov ${ }^{2}$, I. Pogorelsky ${ }^{3}$ and Z. Najmudin ${ }^{1}$ \\ ${ }^{1}$ The John Adams Institute for Accelerator Science, Blackett Laboratory, Imperial College London, \\ London SW7 2BW, UK \\ ${ }^{2}$ Stony Brook University, Stony Brook, NY 11794, USA \\ ${ }^{3}$ Accelerator Test Facility, Brookhaven National Laboratory, Upton, NY 11973, USA
}

(Received 22 September 2015; revised 20 January 2016; accepted 20 January 2016)

We report on the experimental demonstration of a technique to generate steep density gradients in gas-jet targets of interest to laser-plasma ion acceleration. By using an intentional low-energy prepulse, we generated a hydrodynamic blast wave in the gas to shape the target prior to the arrival of an intense $\mathrm{CO}_{2}(\lambda \approx 10 \mu \mathrm{m})$ drive pulse. This technique has been recently shown to facilitate the generation of ion beams by shockwave acceleration (Tresca et al., Phys. Rev. Lett., vol. 115 (9), 2015, 094802). Here, we discuss and introduce a model to understand the generation of these blast waves and discuss in depth the experimental realisation of the technique, supported by hydrodynamics simulations. With appropriate prepulse energy and timing, this blast wave can generate steepened density gradients as short as $l \approx 20 \mu \mathrm{m}(1 / e)$, opening up new possibilities for laser-plasma studies with near-critical gaseous targets.

\section{Introduction}

Laser-plasma particle accelerators can generate bunches of energetic ions with energies $\gg 1 \mathrm{MeV}$ and charge in excess of $10 \mathrm{nC}$ (Daido, Nishiuchi \& Pirozhkov 2012; Macchi, Borghesi \& Passoni 2013). These promising ion sources are being developed for applications in nuclear and medical physics, many of which require control of the beam spectral shape, collimation and charge. Next-generation highpower lasers will run at $10 \mathrm{~Hz}$ and beyond, bringing such applications closer to realisation. However, most research on laser-plasma ion sources has used solid targets, typically metal foils of thickness $\sim 1 \mu \mathrm{m}$. At high repetition rate, using such targets raises significant challenges with debris, target insertion and unwanted secondary radiation such as bremsstrahlung.

Easily replenished, debris-free gaseous targets would alleviate many of these problems. They can generate single-species ion beams, which are challenging for solid targets due to the hydrocarbon layers that typically form on their surface.

$\dagger$ Email address for correspondence: nicholas.dover08@imperial.ac.uk

$\ddagger$ Present address: RadiaSoft LLC, Boulder, CO 80304, USA. 
Previous work has demonstrated longitudinal (along the laser-axis) (Willingale et al. 2006) and transverse (perpendicular to the laser-axis) (Krushelnick et al. 1999; Wei et al. 2004; Sylla et al. 2012; Lifschitz et al. 2014) acceleration of helium ions from targets below the relativistic critical density at which the plasma becomes opaque to the laser $n_{c r}=\bar{\gamma} n_{c}=\bar{\gamma} \epsilon_{0} m_{e} \omega_{L}^{2} / e^{2}$, where $\bar{\gamma}$ is the cycle-averaged electron Lorentz factor, $n_{c}$ is the critical density and $\omega_{L}$ is the laser frequency. However, transverse acceleration creates a divergent expansion of the ions, making it not suitable for high flux applications, whilst longitudinal acceleration from underdense targets has only been reported using laser intensities in excess of $10^{20} \mathrm{~W} \mathrm{~cm}^{-2}$ and longer $\sim 1 \mathrm{ps}$ pulses as the produced longitudinal accelerating fields are low compared to thin foil targets (Willingale et al. 2006).

More recently, the development of high-power $\mathrm{CO}_{2}$ lasers (Haberberger, Tochitsky \& Joshi 2010; Polyanskiy, Pogorelsky \& Yakimenko 2011), for which the wavelength $\lambda_{L} \approx 10 \mu \mathrm{m}$ results in a critical density $n_{c} \approx 10^{19} \mathrm{~cm}^{-3}$, has allowed studies with gaseous targets at densities greater than $n_{c r}$. Using an opaque but near-critical density target combined with a laser pulse of a few ps results in the generation of an electrostatic collisionless shock (Denavit 1992; Silva et al. 2004; Fiuza et al. 2012) capable of accelerating a fraction of the background ions to a velocity twice the speed of the shock, $v_{i}=2 v_{s}$. Initial experiments accelerated protons to energies in excess of $1 \mathrm{MeV}$ with a small energy spread via this technique (Palmer et al. 2011) and subsequently higher ion energies were produced when a longer, more energetic laser pulse drove the acceleration (Haberberger et al. 2012).

Both of these experiments were driven by a train of intense pulses, characteristic of short-pulse $\mathrm{CO}_{2}$ systems. The pulse train played an essential role in creating a suitable plasma density distribution, resulting in enhanced radiation pressure and stronger laser absorption, leading to the generation of an accelerating electrostatic collisionless shock. However, the shot-to-shot variation in the $\mathrm{CO}_{2}$ pulse train meant the density distribution also varied from shot-to-shot, affecting the reproducibility of the ion beam. Recent breakthroughs with $\mathrm{CO}_{2}$ laser technology have allowed the isolation of a single intense pulse, permitting more control over the laser-plasma interaction (Polyanskiy et al. 2011). However, typical gas-jet targets have linear or parabolic density scale-lengths $\gg 100 \mu \mathrm{m}$, too long for the production of quasi-monoenergetic ion beams (Palmer et al. 2014).

It has been shown that secondary laser pulses can be used to 'machine' gas targets. Periodic structures have been created by irradiating gas with a spatially modulated intense laser and waiting for hydrodynamic expansion to create density perturbations in the gas (Pai et al. 2005). A similar set-up was used to dynamically control the length of gas jets for investigating laser wakefield electron acceleration (Hsieh et al. 2006). More recently, Kaganovich et al. (2014) focused a secondary pulse on a foil near the gas flow to drive a hydrodynamic shock into the gas to create density discontinuities.

In this paper, we outline a method for optically shaping a gas target for application to ion acceleration. An all-optical target manipulation system was developed to modify the density profiles of gas targets by using a $\sim 10-100 \mathrm{~mJ}$ prepulse arriving $25 \mathrm{~ns}$ before the main intense pulse. This prepulse is absorbed locally and heats a small region of gas, which subsequently expands and forms a blast wave. By varying the prepulse we were able to decrease the front density scale-lengths down to $\approx 30 \mu \mathrm{m}(1 / e)$. These targets were subsequently used (Tresca et al. 2015) to generate reproducible proton and helium beams with energies up to $E \sim 1 \mathrm{MeV}$.

This paper gives a detailed overview of the generation of a prepulse induced blast wave. In $\S 2$ we discuss analytical scalings of the blast-wave parameters 
and the resultant density profile, and support this with numerical hydrodynamic simulation to explain and predict the blast-wave properties. This is followed by $\$ 3$ demonstrating experimentally the effect of changing the prepulse energy on the plasma density profile. We finally discuss briefly the effects of the density gradients on the laser-plasma interaction and propose an extension to blast-wave shaping of higher-density targets of interest to ion acceleration using higher-intensity near-IR lasers.

\section{Modelling of prepulse-driven blast waves}

A tightly focussed laser pulse of sufficient intensity can induce breakdown of the target gas, forming a plasma which then absorbs energy from the pulse. For sufficiently low plasma temperatures and laser intensities inverse bremsstrahlung heating dominates, in which electrons gain energy from the incident laser field before locally depositing the energy in the plasma via collisions with nearby ions.

The electron-ion collision frequency is given by

$$
v_{e i}=\frac{1}{3(2 \pi)^{3 / 2}} \frac{n_{e} Z e^{4} \ln \Lambda}{\epsilon_{0}^{2} m_{e}^{1 / 2}\left(k_{b} T_{e}[\mathrm{~K}]\right)^{3 / 2}} \approx \frac{2.9 \times 10^{-12} \mathrm{Zn}_{e}\left[\mathrm{~m}^{-3}\right]}{\left(T_{e}[\mathrm{eV}]\right)^{3 / 2}} \ln \Lambda \mathrm{s}^{-1},
$$

where $n_{e}$ is the plasma electron density, $\ln \Lambda$ is the Coulomb logarithm, $Z$ is the atomic number, $k_{b}$ is the Boltzmann constant and $T_{e}$ is the electron temperature. Note that the free electron density depends on the ionisation fraction, which can evolve dynamically as an increasing amount of energy is absorbed in the plasma. The absorption coefficient for inverse bremsstrahlung is given by

$$
K=\frac{\nu_{e i}}{c}\left(\frac{n_{e}}{n_{c}}\right)\left(1-\frac{n_{e}}{n_{c}}\right)^{-1 / 2}
$$

and the corresponding fraction of laser energy thermally absorbed by the plasma, $\eta=$ $E_{a b s} / E_{L}$, is then given by

$$
\eta=1-\exp \left(-\int K \mathrm{~d} x\right)
$$

where the integral is taken along the laser path (Kruer 1988). As laser energy is absorbed, the plasma heats up, reducing the collision frequency and hence the laser absorption. Therefore $\eta$ depends on the target and focusing parameters.

A simplified quantitative model can be introduced for a hydrogen $(Z=1)$ target by neglecting energy loss to ionisation and assuming full ionisation. For an isotropic initial gas density profile, and assuming the breakdown and laser absorption only occur when the laser intensity is at its maximum at the laser focus $\left(-Z_{R} / 2<x<Z_{R} / 2\right.$, where $Z_{R}$ is the Rayleigh range), this can be simplified to $\eta \simeq 1-\exp \left(-K Z_{R}\right)$. In practice the discharge region may differ from this, particularly for larger prepulse energies with intensities exceeding the breakdown threshold away from focus. Assuming uniform heating over a volume bounded by the laser focal spot and $Z_{R}$ and no hydrodynamic expansion, and using (2.3) for the amount of energy absorbed as the laser transmits through the plasma, the plasma temperature is given by

$$
T_{e}(t)[\mathrm{eV}]=\frac{1}{2 n_{e} Z_{R} e} \int_{0}^{t} I_{L}\left(1-\exp \left(-K(t) Z_{R}\right)\right) \mathrm{d} t .
$$

where $I_{L}$ is the laser intensity. 

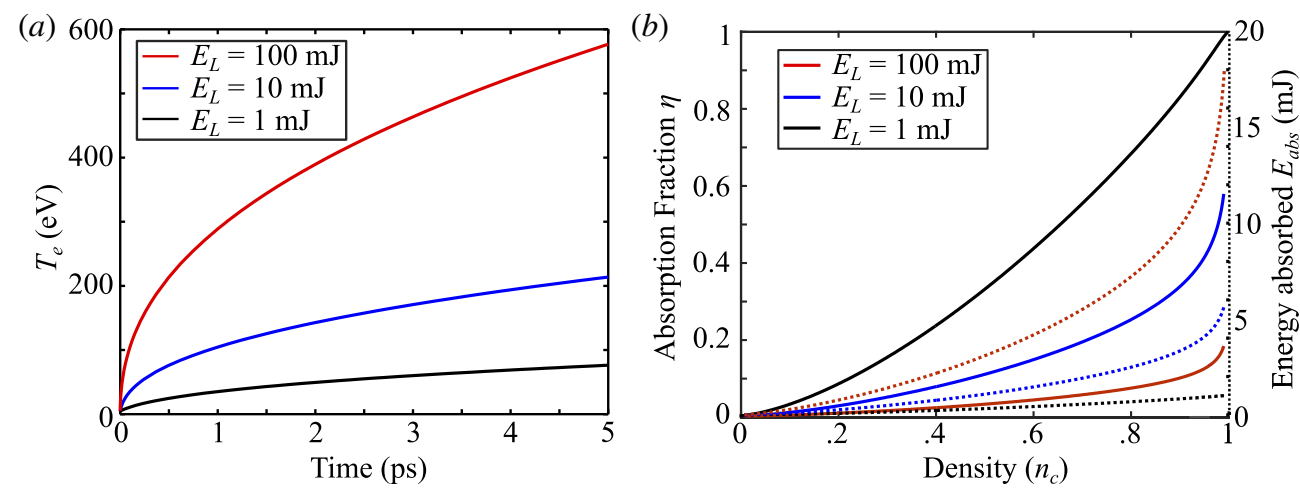

FIgURE 1. (a) Temperature as a function of time for varying laser energy and fixed laser focus $\left(\lambda_{L}=10 \mu \mathrm{m}\right.$, focal spot size $\left.70 \mu \mathrm{m}, Z_{R}=300 \mu \mathrm{m}, \tau_{L}=5 \mathrm{ps}\right)$ and density (hydrogen, $n_{e}=n_{i}=0.5 n_{c}$ ). (b) Absorption fraction of incident laser light $\eta$ (solid lines) and total energy absorbed $E_{a b s}$ (dotted lines) as a function of target density for various laser energies.

In the strongly absorbing limit $\left(K Z_{R} \gg 1\right)$, this gives $T_{e} \propto t$. However, for most of the parameter space considered here, $K Z_{R}<1$. In the very low absorption limit $\left(K Z_{R} \ll 1\right)$ and assuming constant $\ln \Lambda$,

$$
T_{e}(t)[\mathrm{eV}]=\left(\beta t+T_{0}^{5 / 2}\right)^{2 / 5}
$$

where $T_{0}$ is the initial temperature and

$$
\beta \approx 0.0755 \ln \Lambda\left(\frac{n_{e}}{n_{c}}\right)\left(1-\frac{n_{e}}{n_{c}}\right)^{-1 / 2} I_{L}\left[\mathrm{~W} \mathrm{~m}{ }^{-2}\right] \mathrm{eV}^{5 / 2} \mathrm{~s}^{-1}
$$

The approximation required for this analytical solution is not valid for either $n_{e} \approx n_{c}$, nor for $T_{e} \gg T_{0}$, since then $\ln \Lambda$ changes significantly over the prepulse-target interaction. Therefore, to test the largest possible range of parameters we instead integrate (2.4) numerically to model the heating.

Figure 1(a) shows $T_{e}$ as a function of time with $\lambda_{L}=10 \mu \mathrm{m}$, pulse duration $\tau_{L}=5$ ps with a top-hat temporal intensity profile, focal spot size $70 \mu \mathrm{m}$ spot and Rayleigh length $Z_{R}=300 \mu \mathrm{m}$ propagating into a fixed plasma density $n_{e}=n_{i}=$ $5 \times 10^{18} \mathrm{~cm}^{-3}\left(0.5 n_{c}\right.$ for $\left.\lambda=10 \mu \mathrm{m}\right)$ solved with an initial temperature $T_{0}=10 \mathrm{eV}$ for different laser energies. Due to the low initial temperature, the initial absorption fraction is high. The laser energy is efficiently converted into thermal energy and the temperature increases rapidly. However, as the plasma temperature rises, the collision frequency reduces and the rate of laser absorption decreases.

Figure 1(b) shows the absorbed fraction $\eta$ and deposited energy $E_{a b s}$ for different laser energies after irradiation as a function of target density. For a given density, higher energy pulses deposit a smaller fraction of their energy. However, as the absorption coefficient $K$ (2.2) tends to infinity for $n_{e}=n_{c}$, high absorption is recovered by increasing target density, as has been shown experimentally for longer pulses (Mori, Komurasaki \& Arakawa 2004). For sufficiently high initial gas density, if the plasma reaches an ionisation state such that $n_{e}>n_{c}$, the prepulse will cease to transmit through the target and instead be reflected at the critical surface, causing 
the above model to break down. Note that this model neglects breakdown and ionisation; decreasing the prepulse energy below the breakdown threshold will result in no absorption. Furthermore, it considers only collisional heating whereas further absorption may occur from coupling to parametric instabilities or resonances.

Over a 5 ps pulse duration, the hydrodynamic expansion of the plasma is negligible. However, after the initial energy deposition, the heated plasma will expand into the surrounding ambient medium as a collisional blast wave (Sedov 1959; Zel'dovich \& Raizer 1966). A cavity forms surrounded by a dense wall at the expanding shock front. Whilst the blast-wave shock is strongly driven (i.e. the pressure ahead of the shock is negligible compared to the pressure behind the shock), the equations of motion follow a self-similar solution (Sedov 1959). The radius of the blast wave at time $t$ is then given by

$$
r_{b w}(t)=\zeta_{0}(\gamma)\left(\frac{E_{a b s}}{\rho}\right)^{1 /(2+\alpha)} t^{2 /(2+\alpha)},
$$

where $\alpha=2(3)$ is the dimensionality of the shock for a cylindrically (spherically) expanding shock, $\rho$ is the mass density of the background fluid, and $\zeta_{0}(\gamma)$ is a numerical constant $\sim 1$ which is a function of the ratio of specific heats $\gamma$ and the dimensionality $\alpha$ (Sedov 1959). Therefore, the blast-wave size is dependent on the energy absorbed from the laser prepulse, which, as was shown in figure 1, itself varies on target and laser parameters. Although the prepulse may initially heat a cylinder of gas, as $r_{b w} \gg Z_{r}$, the expansion becomes increasingly three dimensional and the expansion is best described as a spherically expanding shock $(\alpha=3)$. Although the above solution is valid for a homogenous target distribution, real gas-jet targets will have density gradients affecting shock propagation. Shockwave expansion in density gradients is also of importance to, for example, supernova explosions in astrophysics (see e.g. review by Ostriker \& McKee (1988)). For example, the well-known Kompaneets equation describes a blast wave expanding into an exponentially varying density profile of the form $\rho(x) \propto \exp (-x / l)$, where $l$ is the exponential scale-length (Kompaneets 1960).

To test arbitrary density profiles and initial deposition-region characteristics, we performed hydrodynamic simulations of the blast-wave expansion using the FLASH hydrodynamics solver in 2-D cylindrical symmetry. Initially, the target was set to be homogenous with density $\rho=8 \times 10^{-5} \mathrm{~g} \mathrm{~cm}^{-3}$ corresponding to a number density $n_{e}=n_{i} \approx 5 \times 10^{19} \mathrm{~cm}^{-3}$, temperature $T_{i}=293 \mathrm{~K}$ and a fixed specific heat ratio $\gamma=1.4$, describing a hydrogen-gas target for laser-plasma interactions. Note that the temperature of gas puffed from a gas jet is likely to be lower than this as it expands into the vacuum, and will depend on the nozzle geometry. However, the counter pressure of the ambient medium is negligible in the conditions simulated, and so does not affect the applicability to experiment. A spherical region with radius $r_{\text {dep }} \approx w_{L}=70 \mu \mathrm{m}$ of fluid was initialised with thermal energy $E_{a b s}$, and simulated for $25 \mathrm{~ns}$.

The heated region quickly expands, within 1 ns forming an evacuated cavity with increasing density around the edge up to a shock front. The blast-wave shock expands into the ambient gas. FLASH features adaptive mesh refinement, AMR, allowing higher resolution around shock-fronts which provides a more accurate simulation of the blast-wave wall. The effect of increasing AMR can be seen in figure 2(a), on a normalised axis together with the self-similar solution (Sedov 1959). Increasing AMR brings the solution closer to the analytical solution, although the finite grid means 

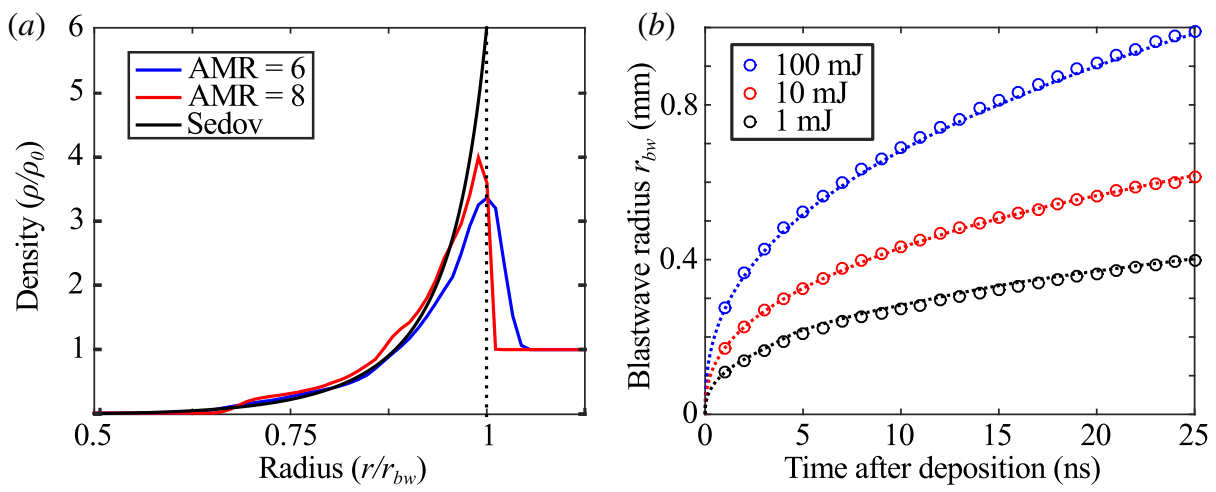

FIGURE 2. (a) Radial density profile of a spherical blast wave from FLASH simulation with $\mathrm{AMR}=6$ (blue) and 8 (red). The black line is the analytical self-similar solution. $(b)$ Simulated blast-wave position as a function of time for different $E_{a b s}$ and fixed density $\rho=8 \times 10^{-5} \mathrm{~g} \mathrm{~cm}^{-3}$, equivalent to $n_{i}=n_{e} \approx 5 \times 10^{19} \mathrm{~cm}^{-3}$ for hydrogen. Dotted lines are analytical fits from (2.7).

the peak density is still slightly lower in the simulation. Indeed, the peak density in the blast wave should reach the strong shock limit,

$$
\rho=\rho_{0} \frac{\gamma+1}{\gamma-1} .
$$

Gases with lower $\gamma$ therefore give higher maximum densities in the blast wave. The width of the shock front, the distance between the peak of the density front and the ambient medium, is determined by the mean free path, and is smaller than the simulation resolution. Figure $2(b)$ shows the blast-wave expansion as a function of time for varying $E_{a b s}$. The analytical fits, calculated from (2.7) for spherical expansion and $\gamma=1.4, \zeta_{0} \approx 1.175^{0.2}$ (Sedov 1959), are also plotted, showing good agreement with the fluid simulation.

For applications to laser-driven shockwave acceleration of ions, a key quantity is the scale-length between the vacuum region and critical density surface. Steeper density gradients allow stronger localised energy deposition and can enhance shockwave generation (Palmer et al. 2014; Tresca et al. 2015). To achieve a steep vacuum-plasma interface, a blast wave can be generated in the gas target close enough to the gas-jet edge that the shock expands into the vacuum region. The gas nozzle used in previous ion-generation experiments with high-intensity $\mathrm{CO}_{2}$ lasers has a gas profile at laser focus height $\approx 1 \mathrm{~mm}$ above the gas-jet exit that is well approximated by a triangle with side lengths $\approx 800 \mu \mathrm{m}$ (Najmudin et al. 2011).

To model the blast-wave expansion for this nozzle, further simulations were performed using this initial gas density profile. The gentle density gradient of the gas jet parallel to the nozzle flow was neglected to allow the use of 2-D cylindrical geometry. The hydrogen gas was initialised as a triangle with a peak density equivalent to $n_{p}=n_{e}=5 \times 10^{19} \mathrm{~cm}^{-3}$, and side length $800 \mu \mathrm{m}$. Outside the gas-jet region, the density was set to be 1000 times lower than $n_{p}$. Note that this provides a counter pressure that is negligible, and approximates the low pressure 'vacuum' outside of the gas jet.

Thermal energy was initially deposited as a sphere with radius $70 \mu \mathrm{m}$ at a position $200 \mu \mathrm{m}$ from the peak density. The subsequent expansion for three different initial 

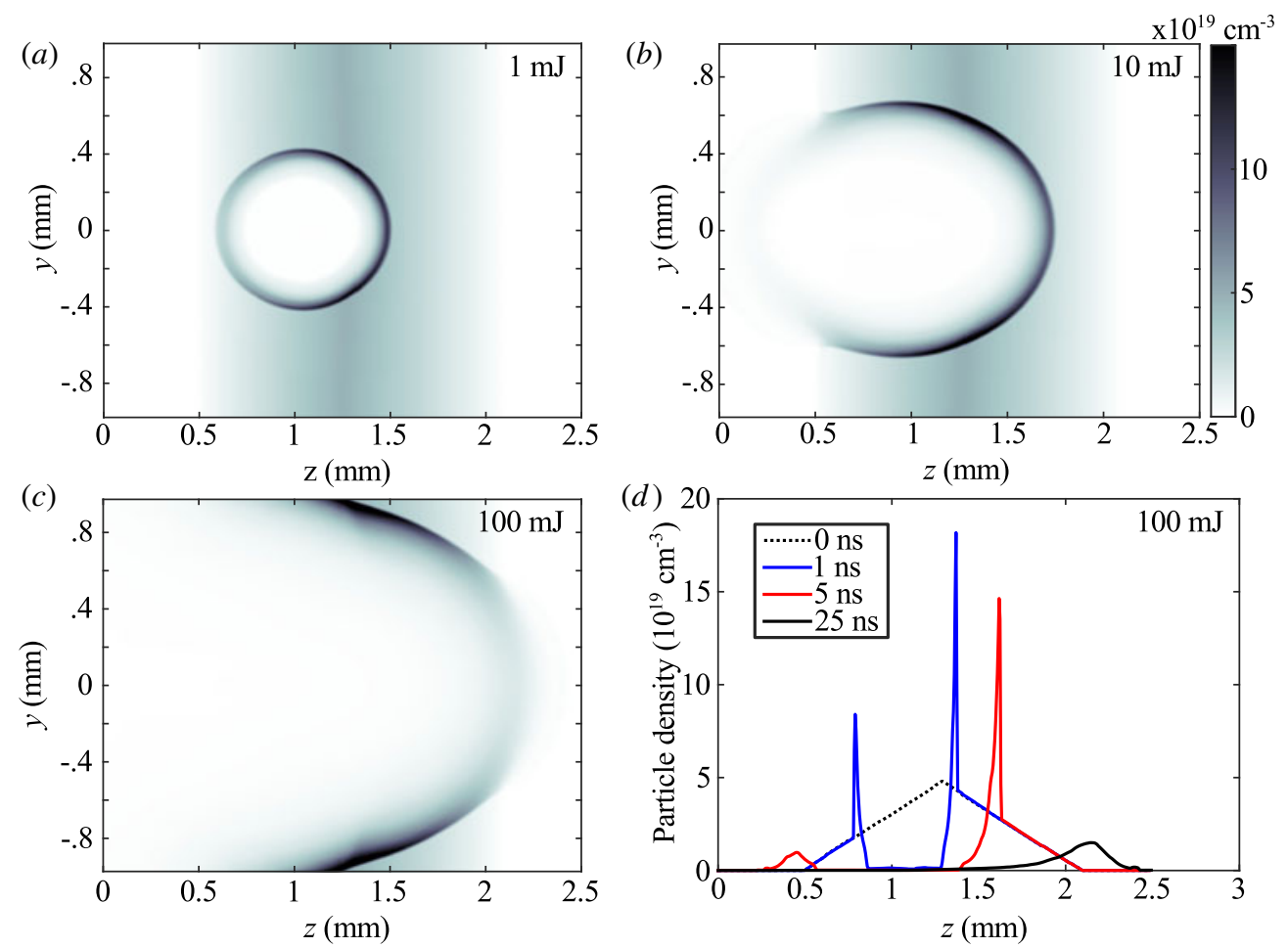

FIGURE 3. $(a-c)$ Gas particle density distribution at $t=25 \mathrm{~ns}$ after energy deposition for $E_{a b s}=1,10,100 \mathrm{~mJ}$ respectively with initial triangular-shaped density profile. $(d)$ The laser-axis $(y=0)$ density distribution at different times in the expansion for $E_{a b s}=100 \mathrm{~mJ}$.

energies is given in figure $3(a-c)$ at $25 \mathrm{~ns}$ after the start of the simulation. For $E_{a b s}=$ $1 \mathrm{~mJ}$, expansion is approximately spherical and the cavity has not expanded into the vacuum region. At $E_{a b s}=10 \mathrm{~mJ}$, the shock slows more quickly in the high-density region than for low density. Hence the blast wave has opened up into the 'vacuum' region, leaving a highly asymmetric cavity. For even higher $E_{a b s}$, the blast wave exits from the rear side of the gas jet (figure $3 c$ ).

The density profile on the laser-axis at different times is given in figure $3(d)$ for a deposited energy of $E_{a b s}=100 \mathrm{~mJ}$. The blast wave produces densities in excess of the initial maximum density, and steep density features in the gas. The steepness of the density ramp $l$ can be estimated by fitting an exponential density profile $\rho=$ $\rho_{\max } \mathrm{e}^{\left(x-x_{0}\right) / l}$ in the region of the shock ramp, and is $22,45,180 \mu \mathrm{m}$ for 1,5 and $25 \mathrm{~ns}$ respectively. Evidently the scale-length increases with increasing time. The asymmetry caused by deposition of energy off the centre of the gas jet results in a time window between the blast wave opening up to vacuum on the down ramp side, and before the density gradient has lengthened too much. Within this time window a high-intensity pulse can interact with a steep density profile and a higher peak density than otherwise available in the gas jet.

\section{Experimental realisation of gas-jet shaping}

To demonstrate blast-wave shaping of gas jets and its effect on ion shockwave acceleration, we performed an experiment using the high-intensity $\mathrm{CO}_{2}$ laser $\left(\lambda_{L}=\right.$ 

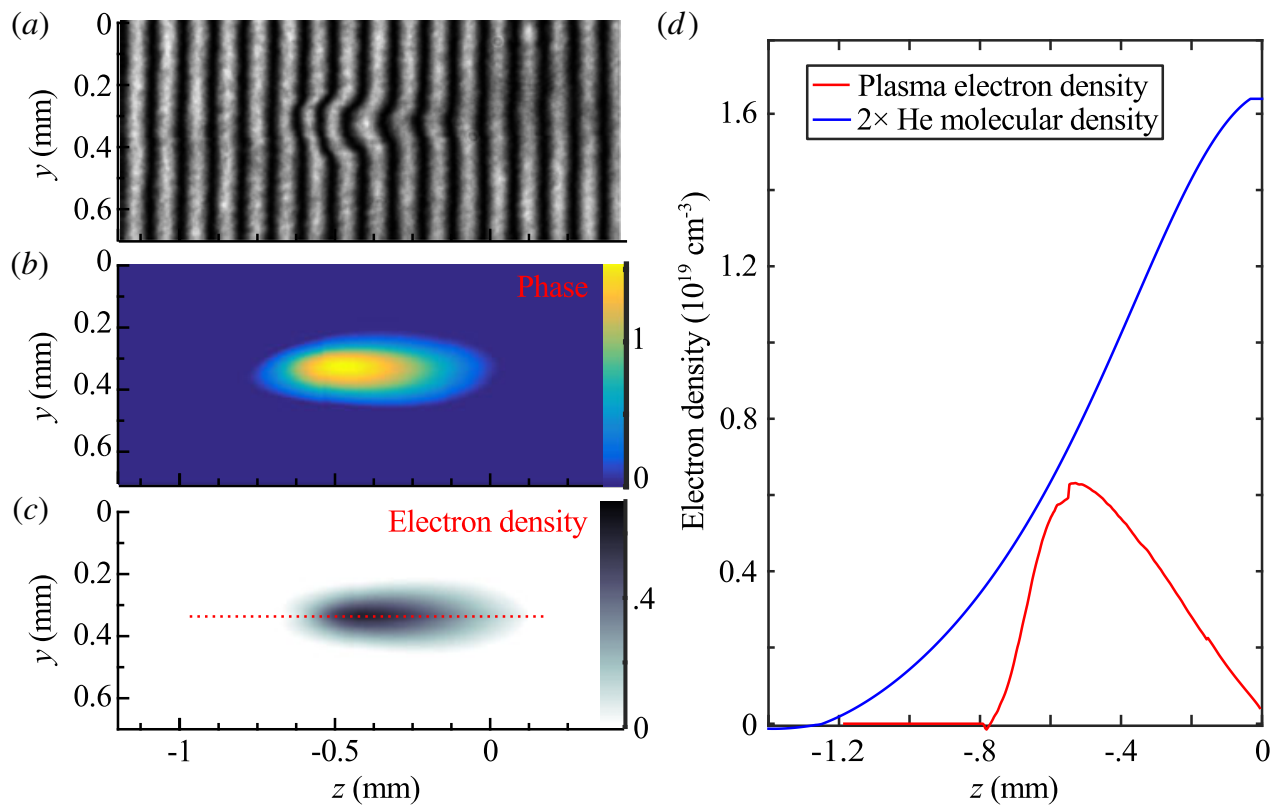

FIGURE 4. $(a-c)$ Raw interferometry, extracted phase (units are radians) and Abel inverted electron-density profile (units are $10^{19} \mathrm{~cm}^{-3}$ ) imaged $250 \mathrm{ps}$ after irradiation of a He target with a $280 \mathrm{~mJ}$ pulse. (d) Line-out along laser-axis of electron density (red) and the neutralgas density (blue, if doubly ionised).

$10.3 \mu \mathrm{m})$ at the Accelerator Test Facility, Brookhaven National Laboratory. A linearly polarised single $\approx 10 \mathrm{~J}$ pulse of duration $\tau_{L} \approx 5$ ps was focused to a spot $w_{0}=65 \mu \mathrm{m}$. This provided a maximal peak intensity $I_{L}=2.5 \times 10^{16} \mathrm{~W} \mathrm{~cm}{ }^{-2}$, corresponding to a normalised vector potential $a_{0}=1.4$. A prepulse with variable energy $\left(\tau_{p p}=5 \mathrm{ps}\right)$ arrived $25 \mathrm{~ns}$ before the main intense pulse. The energy of the prepulse was measured on shot with a photodiode which had been cross-calibrated using a calorimeter. The Rayleigh length of the focal spot was measured to be $Z_{R} \approx 400 \mu \mathrm{m}$.

Helium gas was puffed from a nozzle with either a $0.5 \mathrm{~mm}$ throat, $1 \mathrm{~mm}$ exit diameter (henceforth, referred to as ' $1 \mathrm{~mm}$ '), which gave an approximately triangular profile with side length $800 \mu \mathrm{m}$, or a $1 \mathrm{~mm}$ throat, $2 \mathrm{~mm}$ exit diameter (' $2 \mathrm{~mm}$ '), which featured a $1 \mathrm{~mm}$ side length with $0.8 \mathrm{~mm}$ plateau. Both nozzles could produce peak molecular densities up to $1 \times 10^{20} \mathrm{~cm}^{-3}$ (fully ionised, $n_{e} \leqslant 20 n_{c}$ ) at the laser focal position $1 \mathrm{~mm}$ above the nozzle exit.

The blast-wave formation was measured by a transverse optical probe system, comprising a frequency doubled Nd:YAG pulse $\left(\lambda_{L}=532 \mathrm{~nm}, \tau_{L} \approx 10 \mathrm{ps}\right)$ split into two polarisations and differentially delayed to provide two probing times on a single shot. Each polarisation was simultaneously used for interferometry and shadowgraphy. The absolute timing between the probe pulses and the $\mathrm{CO}_{2}$ pulses was adjustable with an accuracy of \pm 5 ps. The relative timing between the probe beams and main pulse was determined by imaging the rapid plasma formation resulting from the main intense pulse interacting with the gas. A Thomson spectrometer, coupled to a BC-408 polyvinyl-toluene scintillator imaged by an electron multiplying charge coupled device (EMCCD), measured the ion charge state and energy spectrum along the laser-axis.

The prepulse energy deposition characteristics were measured by irradiating the gas target with a single $\approx 280 \mathrm{~mJ}$ pulse. Figure $4(a)$ shows raw interferometry data 

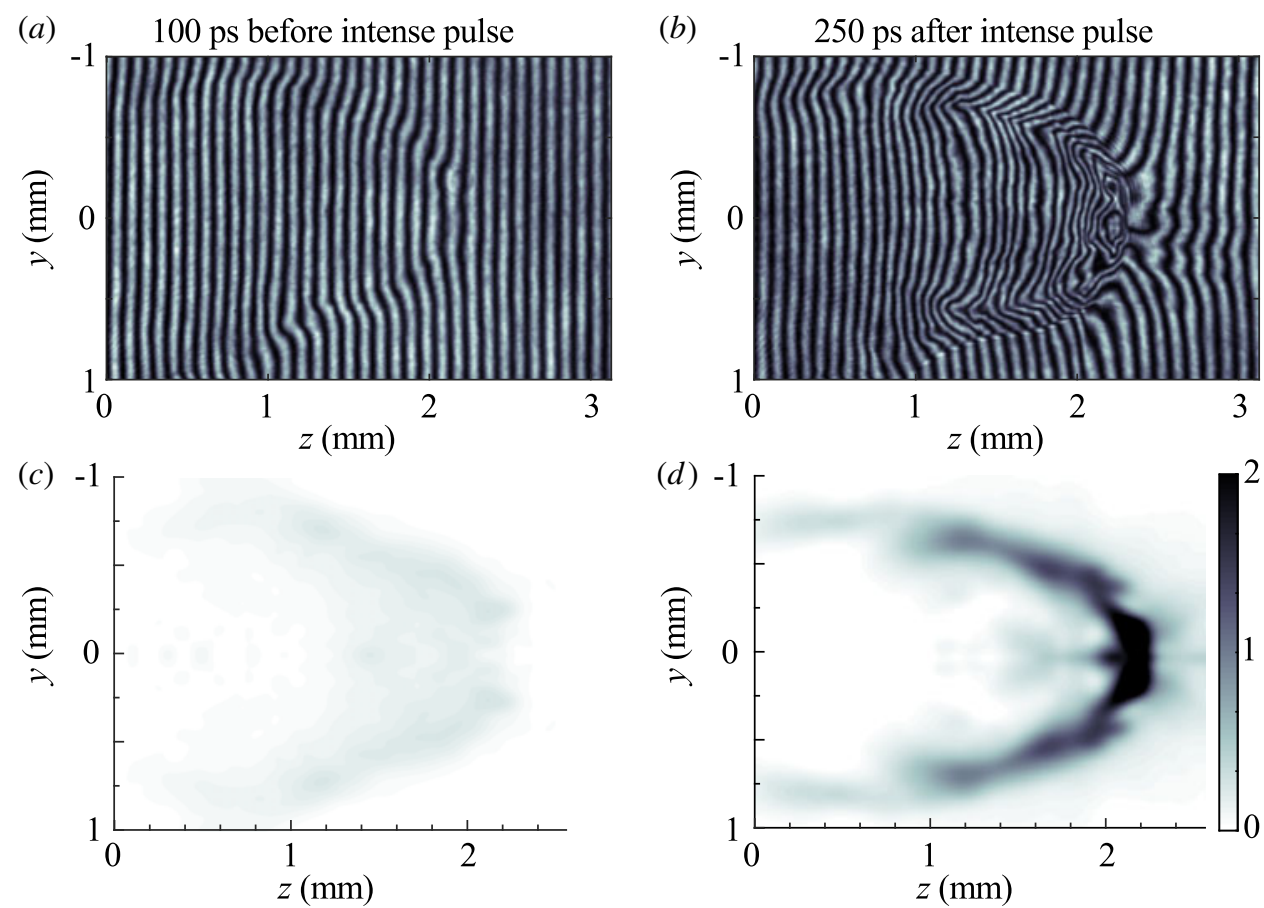

FIGURE 5. $(a, b)$ Raw interferometry 100 ps before and 250 ps after the arrival of the intense pulse, with $E_{p p} \approx 70 \mathrm{~mJ}$ onto a helium plasma with peak density $n_{e}=2.5 n_{c} ;(c, d)$ the corresponding inferred free-electron-density profile.

from the optical probe timed to arrive 250 ps after the initial deposition. Neutral helium gas produces negligible fringe shift compared to the plasma and was therefore ignored. The phase was extracted from interferometry (figure $4 b$ ) and a numerical Abel inversion algorithm was used to calculate the density profile assuming cylindrical symmetry around the laser-axis (figure $4 c$ ). A region of ionised plasma is observed with a transverse full width half maximum $(\mathrm{FWHM}) \approx 100 \mu \mathrm{m}$ and longitudinal FWHM $\approx 400 \mu \mathrm{m}$, similar to the measured focal spot size and Rayleigh range of the laser. A longitudinal line-out along the laser-axis is shown in figure $4(d)$, alongside the fully ionised gas density profile (measured in situ from neutral-gas interferometry). The target is almost fully ionised at the centre of the laser focus. The pulse has evidently deposited some of its energy in a limited region near focus. Following this initial deposition, the heated fluid expands and forms a blast wave as described in the preceding section.

As the main intense pulse arrived 25 ns after the prepulse, probe images were taken on a single shot 100 ps before and 250 ps after the intense interaction to investigate the effects of the target shaping. Interferometry data with prepulse energy $E_{p p} \approx 70 \mathrm{~mJ}$ before and after irradiation of the intense pulse energy $E_{L}=6 \mathrm{~J}$ on the $2 \mathrm{~mm}$ nozzle is shown in figure 5. Before the main pulse a small amount of fringe shift is visible corresponding to a cavity-shaped plasma with peak electron density $\approx 0.3 n_{c}$ (figure $5 c$ ), significantly lower than the original neutral-gas density.

After the intense pulse arrives, a clear blast-wave structure is revealed in the plasma density (figure $5 d$ ) with electron densities in the wall exceeding $3 n_{c}$, greater than the 

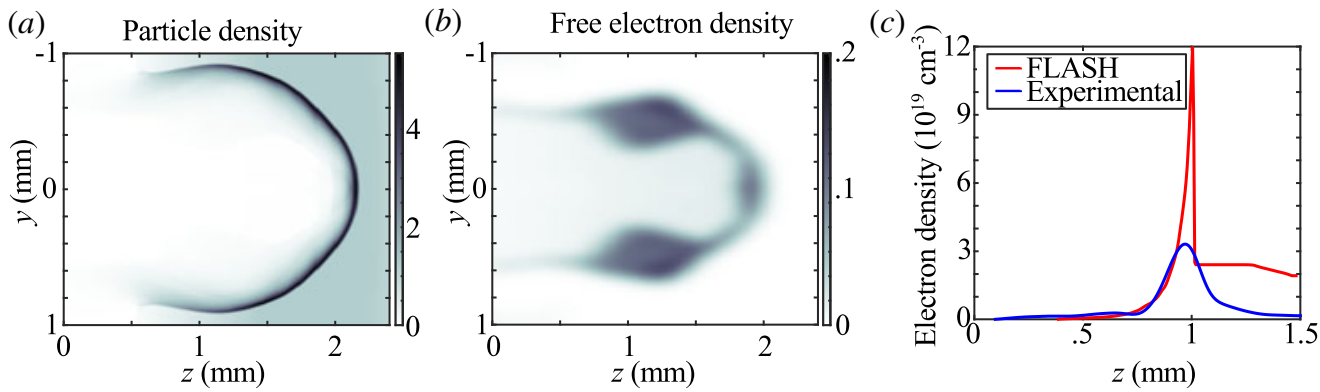

FIgURE 6. (a) Flash simulation: particle density $25 \mathrm{~ns}$ after $50 \mathrm{~mJ}$ is deposited into helium puffed from the $2 \mathrm{~mm}$ gas target, replicating the target conditions in figure 5 ( $2 \mathrm{~mm}$ nozzle, fully ionised peak $n_{e}=2.5 n_{c}$ ), and $(b)$ the estimated free-electron density. (c) laser-axis line-out of the electron density from the FLASH simulation (red, if fully ionised) and experiment (blue).

peak unperturbed initial gas density, $n_{e} \approx 2.5 n_{c}$ if fully ionised. The accuracy of the density measurement is limited by asymmetry in the transverse plane, but the density in the cavity is $<0.25 n_{c}$.

To elucidate these results, FLASH simulations were performed for the $2 \mathrm{~mm}$ helium target, depositing energy in a cylindrical volume of length $400 \mu \mathrm{m}$ and radius $70 \mu \mathrm{m}$, similar to the size of the beam waist and Rayleigh range as well as the size of the ionised region calculated from the data in figure 4. The cylinder was centred at the laser focus position, $200 \mu \mathrm{m}$ in front of the start of the plateau region. Figure 6(a) shows the fluid particle density $25 \mathrm{~ns}$ after depositing $E_{a b s}=50 \mathrm{~mJ}$. A clear cavity has formed, very similar in size and shape to that seen in figure 5 . Prior to the main pulse, the ionisation fraction at this point of the expansion can be estimated by assuming local thermal equilibrium and solving the Saha equation using the temperature and density output from the FLASH simulation. The resulting free-electron density is shown in figure 6. The centre of the cavity is fully ionised due to its high temperature, but the low cavity density means that the resulting phase shift would be small. Approaching the cavity wall, the temperature falls and the free-electron density rapidly drops to zero. The extent of ionisation matches closely to the experimental plasma profile prior to the intense pulse (figure $5 a, c$ ).

The blast wave therefore exists prior to the arrival of the pulse, driven by the prepulse, but is not clearly observed by interferometry due to the low ionisation fraction in the cavity wall as the relative fringe shift from an unionised gas is significantly smaller than that of a plasma of the same density. When the intense pulse arrives, a significant fraction of its energy is deposited into the target causing increased ionisation and further revealing the shock front (figure $5 b, d$ ). Line-outs of the density profile along the laser-axis from the FLASH simulation and the experimental measurement (taken $100 \mu \mathrm{m}$ off-axis to avoid axial numerical effects from the Abel inversion) are shown in figure 6(c). At this stage (250 ps after the interaction), the experimentally measured density scale-length is $l=80 \mu \mathrm{m}$, compared to $l=60 \mu \mathrm{m}$ predicted by the FLASH simulation, with the densest part of the blast wave likely not to be fully ionised. The plasma will also have undergone further expansion after the intense heating by the main intense pulse. Importantly however, the density gradient that the intense laser pulse would interact with has been steepened 


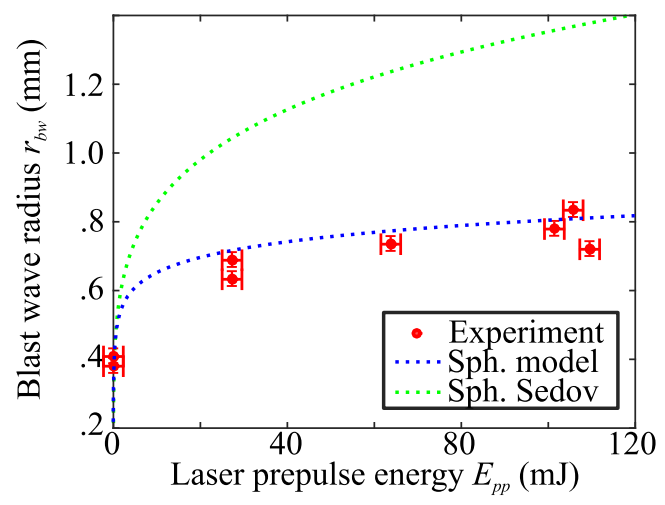

FIGURE 7. Experimental variation of blast-wave radius $250 \mathrm{ps}$ after the main pulse at fixed gas density against $E_{p p}$, together with the analytical Sedov solution where $E_{a b s}=E_{p p}$ (green dotted line), and $E_{a b s}$ modified by the absorption model (blue dotted line). The error on the laser prepulse energy measurement is estimated from noise level on the calibrated photodiode trace.

significantly. As described previously, the scale-length can be shortened by optimising the prepulse energy, and scale-lengths down to $40 \mu \mathrm{m}$ were observed for helium (Tresca et al. 2015).

The effect of experimentally varying $E_{p p}$ on the transverse size of the deposition region at the point of widest expansion (corresponding to an initial density $n_{i} \approx 0.4 n_{c}$ ), while keeping the target conditions constant, is shown in figure 7, measured 250 ps after the main pulse. The transverse size was measured by the location of the sharp intensity variation on shadowgraphy images which indicate the extent of the blast-wave feature. There is a gradual increase in the radius with increasing prepulse energy. If the prepulse energy was fully converted to thermal energy which drives the blast wave (i.e. $E_{p p}=E_{a b s}$ ), the estimated radius for a spherical expansion into a isotropic gas of $n_{i}=0.4 n_{c}$ would be given by the green dotted line (2.7). Despite a cylindrical deposition of laser energy, a spherical expansion model is justified in the late stage as the longitudinal size becomes much larger than the initial deposition length. The model has limited applicability since the target is not isotropic. Nevertheless, the dependence of blast-wave radius $r_{b w}$ on deposited energy $E_{a b s}$ if all of the energy is assumed to be deposited in this volume would still be similar to $r_{b w} \propto\left(E_{a b s}\right)^{0.2}$, which poorly matches the experimental data. Including the energy-deposition model introduced in the previous section, and using the focal spot parameters $\left(w_{0}=70 \mu \mathrm{m}, Z_{R}=400 \mu \mathrm{m}\right)$ and $E_{p p}$ as input, (the dotted blue line) predicts a slower response to increasing prepulse energy. This is due to the energy-absorption fraction diminishing with increased laser energy, following much more closely the experimental observations.

The effect of using prepulse energies outside the optimal range is shown in figure 8, taken using the $1 \mathrm{~mm}$ nozzle with a peak density $n_{i}=0.8 n_{c}$. Due to a lower density at the laser focal position the absorption fraction is smaller, and hence a larger prepulse is required to create a similarly sized cavity to that in figure 5. If the prepulse is too small, only a small cavity can form, and does not open up to the vacuum region (figure $8 a$ ). For such a plasma, the intense pulse arrives and is adversely absorbed in the rising edge of the gas jet before the cavity, and does not interact with a steepened profile. For a very large prepulse (figure $8 b$ ), the blast wave formed propagates too 

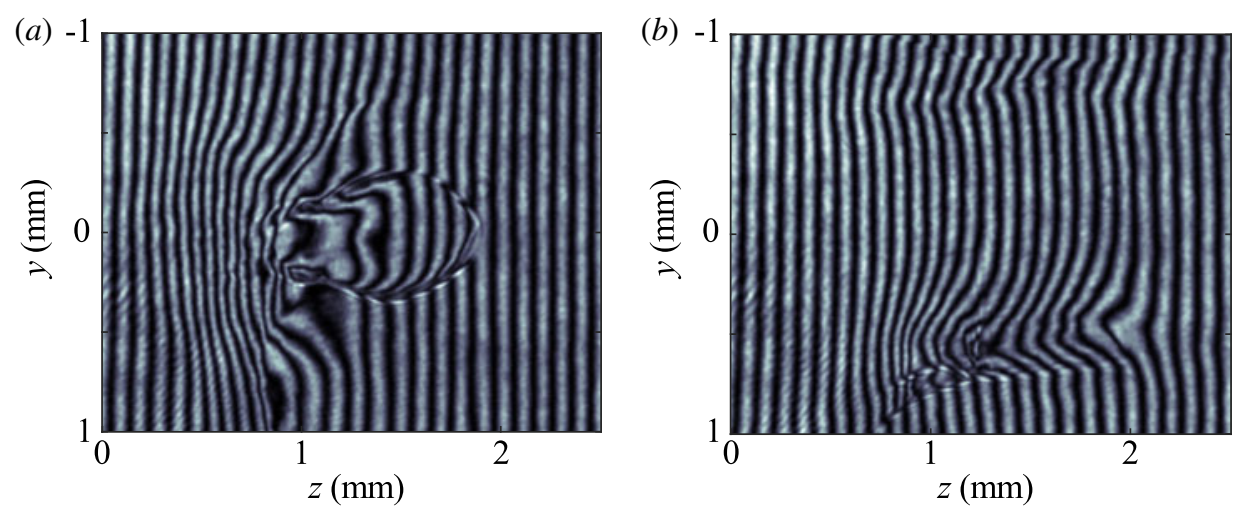

FIGURE 8. Interferometry from $250 \mathrm{ps}$ after the interaction with (a) $E_{p p}=120 \mathrm{~mJ}$ and (b) $E_{p p}=1.6 \mathrm{~J}$.

far and completely blows through the jet, so the intense pulse can only interact with a low-density remnant in the centre of the cylindrical cavity.

The advantages of having a steepened density profile for ion acceleration are described in Tresca et al. (2015). With no prepulse, the intense pulse was absorbed over a long scale-length and no longitudinal ion generation was observed. However, when the prepulse values were $\approx 100-250 \mathrm{~mJ}$ for the $1 \mathrm{~mm}$ nozzle and a peak $n_{e}=1.6 n_{c}$, broadband helium beams were reproducibly generated with maximum observable energies up to $\approx 1.5 \mathrm{MeV}$. When exceedingly large prepulses were used $(>300 \mathrm{~mJ})$, again no ion beams were observed since the density scale-length increases and eventually, as shown in figure $8(b)$, there is no longer an overcritical plasma for the laser to interact with. Particle-in-cell simulations confirmed that using a steepened density profile is essential to generate ion beams from collisionless electrostatic shocks.

Using hydrogen as a target gas also resulted in reproducible broadband beams. However, the lower ratio of specific heat $\gamma \approx 1.4$ provides even steeper cavity walls (Sedov 1959) and a higher peak density (2.8). We note that the prepulse energies required to produce density profiles suitable for ion acceleration were lower for hydrogen than helium. Although increasing $Z$ increases plasma collisionality and hence laser absorption, a larger mass density for a given electron density results in the blast wave still expanding more slowly (2.7) even if the higher $Z$ gas is fully ionised during breakdown. Furthermore, due to its lower binding energy hydrogen requires a lower laser intensity than helium to trigger the initial plasma breakdown, allowing for a longer heating phase (Gibbon 2005). The density scale-length is minimised for a cavity just large enough to open into the vacuum, and it was found that for the steepest density profiles $(\approx 20 \mu \mathrm{m})$, it was possible to generate quasi-monoenergetic beams with $E_{\max }$ up to $1.4 \mathrm{MeV}$ and energy spread as low as $\approx 5 \%$.

\section{Discussion}

The experimental data shown here and in Tresca et al. (2015) were for $\lambda=$ $10 \mu \mathrm{m}$ pulses, in which the required initial densities, $n_{e} \approx 10^{19} \mathrm{~cm}^{-3}$, are easily achievable from standard gas nozzles used widely in high-intensity laser-plasma interactions. However, using high-density gas jets, it would be possible to apply the 

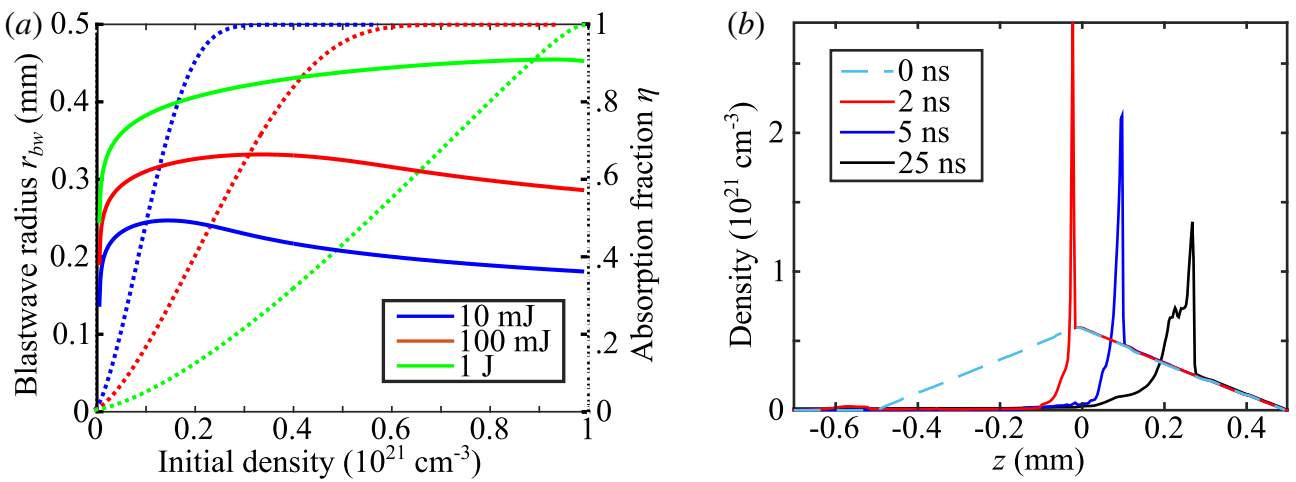

FIGURE 9. (a) Example blast-wave radii achievable for different $E_{a b s}$ after $5 \mathrm{~ns}$ expansion with $\lambda_{L}=1 \mu \mathrm{m}, \tau_{L}=5 \mathrm{ps}, w_{0} \approx 50 \mu \mathrm{m}$ heating a volume length $300 \mu \mathrm{m}$ in an initially uniform plasma (solid, left $y$-axis), and the corresponding absorption fraction (dotted, right $y$-axis). (b) Axial density profiles at different times from FLASH simulations of blast waves generated in a high-density gas jet.

same techniques to create steepened profiles which are overdense to near-infrared wavelengths $\left(\lambda_{L} \approx 1 \mu \mathrm{m}\right)$. The higher collisionality results in a higher prepulse absorption efficiency, $\eta$. Despite the higher density requirements and $r_{b w} \propto\left(E_{a b s} / n_{i}\right)$ scaling, it is still possible to generate blast waves of radii of the same order as a typical sonic gas jet using $E_{p p}<1 \mathrm{~J}$. This is shown in figure $9(a)$ in which the output of the numerical model (2.4) is used to estimate the blast-wave size using (2.7). Axial density profiles from FLASH simulations using a typical gas density profile from Sylla et al. (2012) with peak hydrogen density $n_{i}=0.7 \times 10^{21} \mathrm{~cm}^{3}$ are shown at various times for $E_{a b s}=100 \mathrm{~mJ}$ deposited in a cylinder radius $50 \mu \mathrm{m}$ and length $200 \mu \mathrm{m}$, centred $250 \mu \mathrm{m}$ from peak density (figure $9 \mathrm{~b}$ ), demonstrating density profile steepening. Furthermore, the increase in the peak density from the shockwave helps to relax the requirements on high gas-jet backing pressure for achieving $n_{e}>n_{c r}$, as necessitated for an opaque target.

We have therefore demonstrated all-optical tailoring of gas-jet profiles of interest to longitudinal radiation pressure driven ion acceleration mechanisms. By carefully choosing an appropriate prepulse, we have driven hydrodynamic blast waves to produce targets with significantly steeper density gradients than a typical gas jet. This scheme has been successfully used to generate longitudinal ion beams via collisionless shock acceleration (Tresca et al. 2015), and opens up new possibilities for intense laser interactions with near-critical density targets.

\section{Acknowledgements}

This work was supported by the US DOE grant DE-FG02- 07ER41488, UK EPSRC grant EP/K022415/1 and STFC grant ST/J002062/1. FLASH was developed by the DOE NNSA ASC and NSF-supported FCCS at the University of Chicago. EPOCH development was supported by EPSRC grants EP/G054940/1, EP/G055165/1 and EP/G056803/1. Computing resources were provided by Imperial College HPC services. 


\section{REFERENCES}

Daido, H., Nishiuchi, M. \& Pirozhkov, A. S. 2012 Review of laser-driven ion sources and their applications. Rep. Prog. Phys. 75 (5), 056401.

Denavit, J. 1992 Absorption of high-intensity subpicosecond lasers on solid density targets. Phys. Rev. Lett. 69 (21), 3052-3055.

Fiuza, F., Stockem, A., Boella, E., Fonseca, R., Silva, L., Haberberger, D., Tochitsky, S., Gong, C., Mori, W. \& JoshI, C. 2012 Laser-driven shock acceleration of monoenergetic ion beams. Phys. Rev. Lett. 109 (21), 215001.

GibBon, P. 2005 Short Pulse Laser Interactions with Matter, 1st edn. Imperial College Press.

Haberberger, D., Tochitsky, S., Fiuza, F., Gong, C., Fonseca, R. A., Silva, L. O., Mori, W. B. \& JoshI, C. 2012 Collisionless shocks in laser-produced plasma generate monoenergetic high-energy proton beams. Nat. Phys. 8 (1), 95-99.

Haberberger, D., Tochitsky, S. \& Joshi, C. 2010 Fifteen terawatt picosecond $\mathrm{CO}_{2}$ laser system. Opt. Express 18 (17), 17865-17875.

Hsieh, C. T., Huang, C. M., Chang, C. L., Ho, Y. C., Chen, Y. S., Lin, J. Y., Wang, J. \& CHEN, S. Y. 2006 Tomography of injection and acceleration of monoenergetic electrons in a laser-wakefield accelerator. Phys. Rev. Lett. 96 (9), 7-10.

Kaganovich, D., Gordon, D. F., Helle, M. H. \& Ting, A. 2014 Shaping gas jet plasma density profile by laser generated shock waves. J. Appl. Phys. 116 (1), 013304.

Kompaneets, A. S. 1960 A point explosion in an inhomogeneous atmosphere. Sov. Phys. Dokl. 5, $46-48$.

Kruer, W. 1988 The Physics of Laser Plasma Interaction. Addison-Wesley.

Krushelnick, K., Clark, E. L., Najmudin, Z., Salvati, M., Santala, M. I. K., Tatarakis, M., Dangor, A. E., Malka, V., Neely, D., Allott, R. et al. 1999 Multi-MeV ion production from high-intensity laser interactions with underdense plasmas. Phys. Rev. Lett. 83 (4), 737-740.

Lifschitz, A., Sylla, F., Kahaly, S., Flacco, A., Veltcheva, M., Sanchez-Arriaga, G., LefebVRe, E. \& MALKA, V. 2014 Ion acceleration in underdense plasmas by ultra-short laser pulses. New J. Phys. 16 (3), 033031.

Macchi, A., Borghesi, M. \& PASsOni, M. 2013 Ion acceleration by superintense laser-plasma interaction. Rev. Mod. Phys. 85 (2), 751-793.

Mori, K., Komurasaki, K. \& Arakawa, Y. 2004 Energy transfer from a laser pulse to a blast wave in reduced-pressure air atmospheres. J. Appl. Phys. 95 (11), 5979-5983.

Najmudin, Z., Palmer, C. A. J., Dover, N. P., Pogorelsky, I. V., Babzien, M., Dangor, A. E., Dudnikova, G. I., Foster, P. S., Green, J. S., Ispiriyan, M. et al. 2011 Observation of impurity free monoenergetic proton beams from the interaction of a $\mathrm{CO}_{2}$ laser with a gaseous target. Phys. Plasmas 18 (5), 056705.

Ostriker, J. P. \& McKee, C. F. 1988 Astrophysical blastwaves. Rev. Mod. Phys. 60 (1), 1-68.

Pai, C. H., Huang, S. Y., Kuo, C. C., Lin, M. W., Wang, J., Chen, S. Y., Lee, C. H. \& Lin, J. Y. 2005 Fabrication of spatial transient-density structures as high-field plasma photonic devices. Phys. Plasmas 12 (7), 1-4.

Palmer, C. A. J., Dover, N. P., Pogorelsky, I. V., Babzien, M., Dudnikova, G. I., Ispiriyan, M., Polyanskiy, M. N., Schreiber, J., ShKolnikov, P., YAKimenko, V. et al. 2011 Monoenergetic proton beams accelerated by a radiation pressure driven shock. Phys. Rev. Lett. 106 (1), 014801.

Palmer, C. A. J., Dover, N. P., Pogorelsky, I. V., Streeter, M. J. V. \& Najmudin, Z. 2014 Manipulation of laser-generated energetic proton spectra in near critical density plasma. J. Plasma Phys. 1-9.

Polyanskiy, M. N., Pogorelsky, I. V. \& Yakimenko, V. 2011 Picosecond pulse amplification in isotopic $\mathrm{CO}_{2}$ active medium. Opt. Express 19 (8), 7717-7725.

Sedov, L. I. 1959 Similarity and Dimensional Methods in Mechanics. Academic.

Silva, L. O., Marti, M., Davies, J. R., Fonseca, R. A., Ren, C. \& Mori, W. B. 2004 Proton shock acceleration in laser-plasma interactions. Phys. Rev. Lett. 92 (1), 015002. 
Sylla, F., Veltcheva, M., Kahaly, S., Flacco, A. \& Malka, V. 2012 Development and characterization of very dense submillimetric gas jets for laser-plasma interaction. Rev. Sci. Instrum. 83 (3), 033507.

Tresca, O., Dover, N. P., Cook, N., Maharjan, C., Polyanskiy, M. N., Najmudin, Z., Shkolnikov, P. \& Pogorelsky, I. V. 2015 Spectral modification of shock accelerated ions using a hydrodynamically shaped gas target. Phys. Rev. Lett. 115 (9), 094802.

Wei, M. S., Mangles, S. P. D., Najmudin, Z., Walton, B., Gopal, A., Tatarakis, M., Dangor, A. E., Clark, E. L., Evans, R. G., Fritzler, S. et al. 2004 Ion acceleration by collisionless shocks in high-intensity-laser-underdense-plasma interaction. Phys. Rev. Lett. 93 (15), 155003.

Willingale, L., Mangles, S. P. D., Nilson, P. M., Clarke, R. J., Dangor, A. E., Kaluza, M. C., Karsch, S., Lancaster, K. L., Mori, W. B., Najmudin, Z. et al. 2006 Collimated multi-MeV ion beams from high-intensity laser interactions with underdense plasma. Phys. Rev. Lett. 96 (24), 245002.

Zel'dovich, YA. B. \& RAIZer, Yu. P. 1966 Physics of Shock Waves and High-Temperature Hydrodynamic Phenomena. Dover. 